University of Nebraska - Lincoln

DigitalCommons@University of Nebraska - Lincoln

10-1-2006

\title{
Structures and relative stability of neutral gold clusters: $\mathrm{Au}_{n}$
} $(n=15-19)$

\author{
Satya S. Bulusu \\ University of Nebraska-Lincoln, sbulusu@iiti.ac.in \\ Xiao Cheng Zeng \\ University of Nebraska-Lincoln, xzeng1@unl.edu
}

Follow this and additional works at: https://digitalcommons.unl.edu/chemzeng

Part of the Chemistry Commons

Bulusu, Satya S. and Zeng, Xiao Cheng, "Structures and relative stability of neutral gold clusters: Aun (n=15-19)" (2006). Xiao Cheng Zeng Publications. 2.

https://digitalcommons.unl.edu/chemzeng/2

This Article is brought to you for free and open access by the Published Research - Department of Chemistry at DigitalCommons@University of Nebraska - Lincoln. It has been accepted for inclusion in Xiao Cheng Zeng Publications by an authorized administrator of DigitalCommons@University of Nebraska - Lincoln. 


\title{
Structures and relative stability of neutral gold clusters: $\mathrm{Au}_{n}(n=15-19)$
}

\author{
Satya Bulusu and X. C. Zeng a) \\ Department of Chemistry, University of Nebraska-Lincoln, Lincoln, Nebraska 68588 and Nebraska Center \\ for Materials and Nanoscience, University of Nebraska-Lincoln, Lincoln, Nebraska 68588
}

(Received 21 June 2006; accepted 15 August 2006; published online 17 October 2006)

\begin{abstract}
We performed a global-minimum search for low-lying neutral clusters $\left(\mathrm{Au}_{n}\right)$ in the size range of $n=15-19$ by means of basin-hopping method coupled with density functional theory calculation. Leading candidates for the lowest-energy clusters are identified, including four for $\mathrm{Au}_{15}$, two for $\mathrm{Au}_{16}$, three for $\mathrm{Au}_{17}$, five for $\mathrm{Au}_{18}$, and one for $\mathrm{Au}_{19}$. For $\mathrm{Au}_{15}$ and $\mathrm{Au}_{16}$ we find that the shell-like flat-cage structures dominate the population of low-lying clusters, while for $\mathrm{Au}_{17}$ and $\mathrm{Au}_{18}$ spherical-like hollow-cage structures dominate the low-lying population. The transition from flat-cage to hollow-cage structure is at $\mathrm{Au}_{17}$ for neutral gold clusters, in contrast to the anion counterparts for which the structural transition is at $\mathrm{Au}_{16}^{-}[\mathrm{S}$. Bulusu et al., Proc. Natl. Acad. Sci. U.S.A. 103, 8362 (2006)]. Moreover, the structural transition from hollow-cage to pyramidal structure occurs at $\mathrm{Au}_{19}$. The lowest-energy hollow-cage structure of $\mathrm{Au}_{17}$ (with $C_{2 v}$ point-group symmetry) shows distinct stability, either in neutral or in anionic form. The distinct stability of the hollow-cage $\mathrm{Au}_{17}$ calls for the possibility of synthesizing highly stable core/shell bimetallic clusters $\mathrm{M} @ \mathrm{Au}_{17}(\mathrm{M}=$ group I metal elements). (C) 2006 American Institute of Physics.
\end{abstract}

[DOI: $10.1063 / 1.2352755]$

\section{INTRODUCTION}

Gold clusters and nanoparticles hold great promise for applications in catalysis, medical sciences, and sensors. ${ }^{1-5}$ Experimental and theoretical investigations have shown that gold clusters exhibit some unique properties such as strong relativistic effect and aurophilic attraction. ${ }^{4}$ The strong relativistic effect coupled with the involvement of $d$ orbitals leads to reduced $5 d-6 s$ energy gap as well as strong directional covalent bonds in gold clusters. As a result, gold clusters up to the size $\mathrm{Au}_{20}$ have been shown to exhibit a variety of structures, including two-dimensional (2D) planar, shelllike "flat cage," spherical-like "hollow cage," and pyramidal. $^{6-18}$ In contrast, clusters of $\mathrm{Cu}$ and $\mathrm{Ag}$ only show planar and spherical-like compact structures in the same size range. ${ }^{19-24}$ For neutral gold clusters, $\mathrm{Au}_{n}$, previous high-level $a b$ initio calculations show that a structural transition from 2D planar to three-dimensional (3D) structures occurs within the size range $n=8-10 .{ }^{13,14,17}$ For $n \geqslant 15$, Doye and Wales ${ }^{25}$ performed the first global-minimum search of the lowestenergy clusters using Sutton-Chen potential of gold. They predicted that many low-lying neutral gold clusters favor compact structures. Another early study by Garzon and co-workers ${ }^{26}$ showed that the low-lying neutral gold clusters with sizes $n=19,38$, and 55 adopt amorphouslike compact structures. Note, however, that these early theoretical results were all based on empirical potentials of gold, in which the relativistic effect was not explicitly included.

Later, using a genetic-algorithm global optimization method coupled with tight-binding model and densityfunctional theory (DFT) total-energy calculation (considering relativistic effects), Wang et al. ${ }^{7}$ found a shell-like flatcage lowest-energy structure for $n=15$, and compact

${ }^{a)}$ Electronic mail: xczeng@phase2.unl.edu structures for $n=16-19$. The compact clusters typically consist of an inner core atom and outer "surface" atoms. In another DFT study ${ }^{16} \mathrm{Fa}$ et al. showed that the lowest-energy structure of $n=15$ exhibits shell-like flat-cage structure and that the low-lying structures of $n=16-19$ can be obtained by removing four, three, two, and one corner atoms of the pyramid $\mathrm{Au}_{20}{ }^{10}{ }^{10}$ respectively. After geometric reoptimization, the obtained clusters exhibit hollow-cage-like structures except $n=19$. We have recently carried out a joint experimental/ theoretical study of anion gold clusters. We found that the predominant population of low-lying anion clusters for $n$ $=16-18$ exhibits hollow-cage structures. The transition from the shell-like flat-cage to spherical-like hollow-cage structure occurs at $n=16$ for anion gold clusters. It is known that anion and neutral clusters often do not have the same lowestenergy structure, and thus do not show structural transition at the same size. For example, previous experimental/ theoretical studies showed that the 2D-to-3D transition occurs at $n=12-13$ for anion clusters. ${ }^{8,27}$ Olson et al. ${ }^{13}$ used high-level $a b$ initio coupled-cluster method to evaluate relative stability of $2 \mathrm{D}$ versus $3 \mathrm{D}$ low-lying neutral cluster of $\mathrm{Au}_{8}$. It was found that 3D neutral clusters are lower in energy than the 2D neutral clusters. We also performed coupled-cluster calculation for low-lying clusters of $\mathrm{Au}_{9}$ and $\mathrm{Au}_{10}$ (Ref. 17) and found that 3D neutral clusters are lower in energy than the 2D neutral clusters, consistent with previous finding for $\mathrm{Au}_{8} \cdot{ }^{13}$ The aim of this article is to search for candidates of the lowest-energy neutral clusters in the size range of $n=15-19$. We used the basin-hopping global optimization method ${ }^{25,28}$ directly coupled with DFT total-energy calculation to generate a population of low-lying neutral clusters in the size range of $n=15-19$. We examined the cluster size at which the structural transition from the shelllike flat-cage to spherical-like hollow-cage structure occurs. 


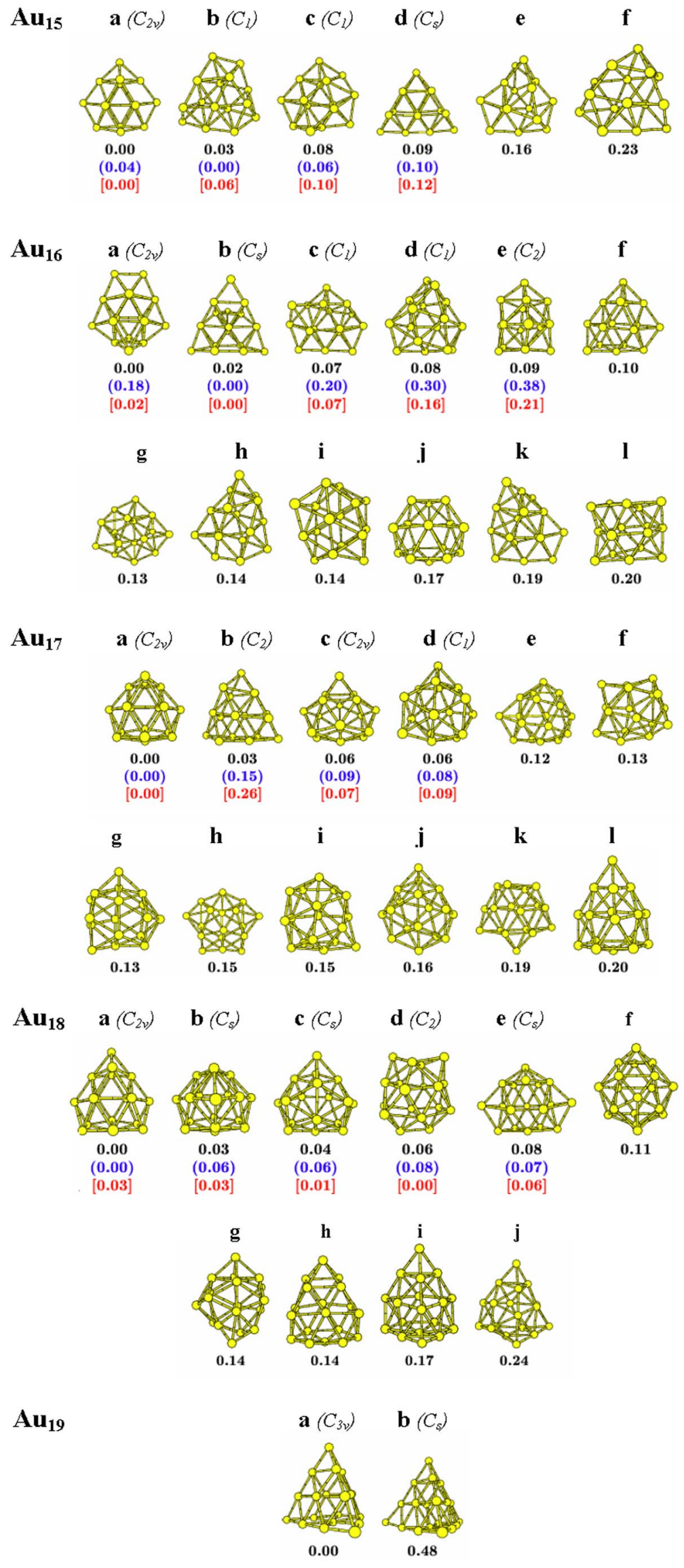

FIG. 1. (Color online) Structures of candidates for the lowest-energy cluster of $\mathrm{Au}_{15}-\mathrm{Au}_{19}$. The energy values (in $\mathrm{eV}$ ) beneath each isomer are the relative energy with respect to the lowest-lying isomer calculated at the corresponding level of DFT. The energy values in black color are based on the PBE/DNP level of theory (Ref. 32). The energy values in parentheses/blue color are based on the PBEPBE/LANL2DZ level of theory (Ref. 33), while the energy values in bracket/red color are based on the single-point-energy calculation at the PBEPBE/SDD+Au(2f)//PBEPBE/LANL2DZ level of theory (Ref. 33). 
TABLE I. Electronic energies (in a.u.) of the leading candidates for the lowest-energy neutral gold clusters $\left(\mathrm{Au}_{15}-\mathrm{Au}_{18}\right)$ calculated at PBEPBE/ LANL2DZ and PBEPBE/SDD+Au( $2 f)$ levels of theory. The boldfaced energy values highlight the lowest-energy isomers which can be dependent on the level of theory.

\begin{tabular}{|c|c|c|}
\hline Cluster & $\begin{array}{l}\text { PBEPBE/LANL2DZ// } \\
\text { PBEPBE/LANL2DZ } \\
\text { (in a.u.) }\end{array}$ & $\begin{array}{c}\text { PBEPBE/SDD }+\mathrm{Au}(2 f) / / \\
\text { PBEPBE/LANL2DZ } \\
\text { (in a.u.) }\end{array}$ \\
\hline $15 a$ & -2032.4117479 & -2037.2872821 \\
\hline $15 b$ & -2032.4131932 & -2037.2849529 \\
\hline $15 \mathrm{c}$ & -2032.4110901 & -2037.2836765 \\
\hline 15d & -2032.4095997 & -2037.2829316 \\
\hline $16 \mathrm{a}$ & -2167.9278636 & -2173.1276271 \\
\hline $16 b$ & -2167.9345715 & -2173.1284951 \\
\hline $16 \mathrm{c}$ & -2167.9271804 & -2173.1260593 \\
\hline $16 d$ & -2167.9235407 & -2173.1225815 \\
\hline $16 \mathrm{e}$ & -2167.9206040 & -2173.1207584 \\
\hline $17 \mathbf{a}$ & -2303.4349226 & -2308.9659302 \\
\hline $17 b$ & -2303.4292864 & -2308.9562466 \\
\hline $17 \mathrm{c}$ & -2303.4316591 & -2308.9635304 \\
\hline 17d & -2303.4321244 & -2308.9625921 \\
\hline $18 \mathbf{a}$ & -2438.9575639 & -2444.8121523 \\
\hline $18 b$ & -2438.9553534 & -2444.8121698 \\
\hline $18 \mathrm{c}$ & -2438.9553769 & -2444.8130040 \\
\hline 18d & -2438.9546772 & -2444.8133062 \\
\hline $18 \mathrm{e}$ & -2438.9551679 & -2444.8111470 \\
\hline
\end{tabular}

Au16

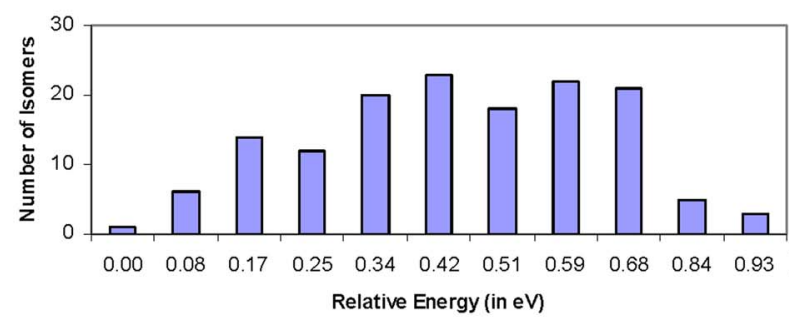

$\mathrm{Au}_{17}$

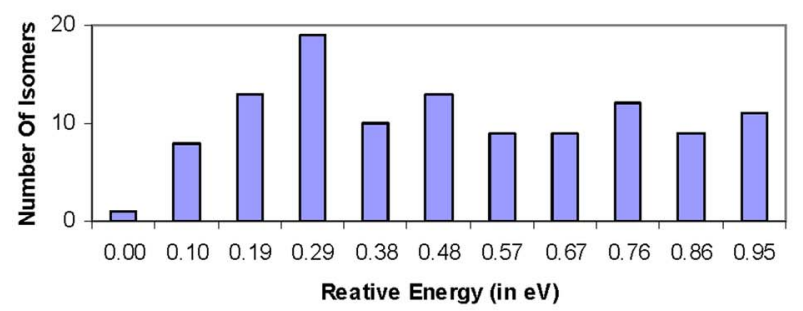

$\mathrm{Au}_{18}$

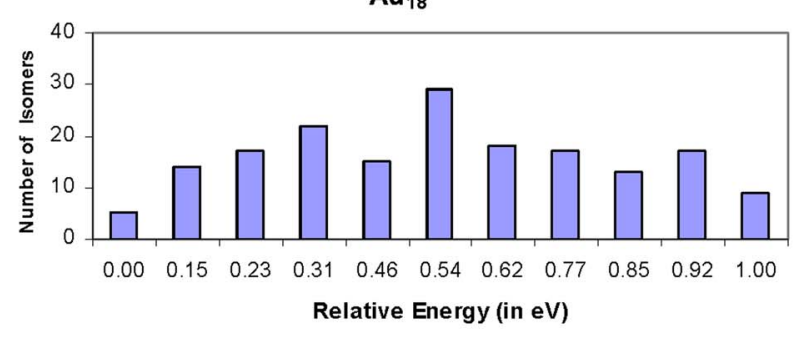

FIG. 2. (Color online) Histograms of the number of low-energy isomers vs the relative energies (with respect to the lowest-energy isomer calculated at the PBE/DNT level of theory) for $\mathrm{Au}_{16}, \mathrm{Au}_{17}$, and $\mathrm{Au}_{18}$. The range of relative energy is set from 0 to $1 \mathrm{eV}$.

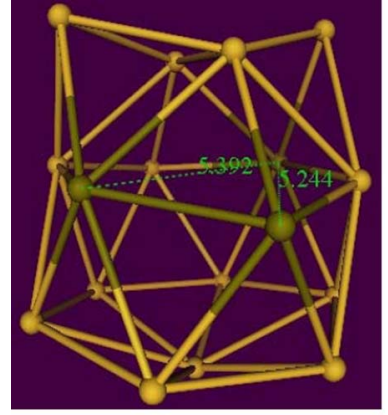

$\mathbf{A u}_{17 \mathbf{a}}$

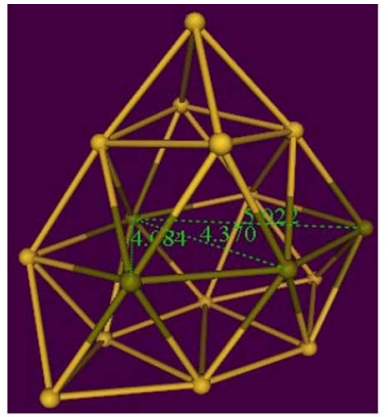

$\mathbf{A} \mathbf{u}_{17 \mathrm{~b}}$
FIG. 3. (Color online) A typical spherical-like hollow-cage structure $\left(\mathrm{Au}_{17 a}\right)$ and shell-like flat-cage structure $\left(\mathrm{Au}_{17 b}\right)$. A flat cage is defined such that besides the overall shape of the cluster is shell-like (or oblate in shape), the $\mathrm{Au}-\mathrm{Au}$ lines (green lines) connecting through the central region of the cage (in the short-axis direction) are in the range of 3-4.5. . Thus, a flat cage may accommodate a small atom such as a hydrogen atom but cannot accommodate another gold atom without major structural distortion. A hollow cage is defined such that the $\mathrm{Au}-\mathrm{Au}$ lines (green lines) connecting through the central region of the cage are all greater than $5.2 \AA$. Thus the hollow cage can accommodate another gold atom.

\section{COMPUTATION METHODS}

We performed a global-minimum search for the lowestenergy gold clusters in the size range of $n=15-19$. We employed the basin-hopping method coupled with (relativistic) density-functional theory calculation. This combined basinhopping/DFT computational approach has been previously used to search for low-lying silicon clusters, ${ }^{29}$ anionic gold clusters, ${ }^{18}$ and bimetallic mixed clusters. ${ }^{30}$

In the basin-hopping search, we typically used 300-500 basin-hopping steps to generate at least 200 structurally different low-energy isomers for each size. We then identified those low-lying isomers whose energy value is within $0.2 \mathrm{eV}$ from the lowest-lying isomer. These low-lying isomers are all regarded as candidates for the lowest-energy structure. This is because the DFT total-energy calculation entails certain intrinsic error bar for small-sized gold clusters. ${ }^{13,14,17} \mathrm{We}$ noticed that for topologically similar clusters, the error bar is relatively smaller, about $0.1 \mathrm{eV}$, whereas for topologically very different clusters, the error bar can be as large as a few tenths of $\mathrm{eV}^{13,17}$ In the DFT calculation, we employed the Perdew-Burke-Ezerhof (PBE) exchange-correlation functional ${ }^{31}$ and double numerical polarized (DNP) basis set implemented in the DMOL3 code. ${ }^{32}$ To examine basis-set effects on the relative energies, we further evaluated the relative energies among the low-lying isomers whose energy is within $0.1 \mathrm{eV}$ from the lowest-lying one, by using a relativistic basis set (LANL2DZ) as well as a very large [SDD $+\mathrm{Au}(2 f)]$ basis set, respectively. The relative-energy values shown in parentheses and blue color (Fig. 1) are based on the optimization with the PBEPBE/LANL2DZ functional/basis set, while the relative-energy values shown in bracket and red color (Fig. 1) are based on the single-point-energy calculation at the PBEPBE/SDD $+\mathrm{Au}(2 f) \| \mathrm{PBEPBE} / \mathrm{LANL} 2 \mathrm{DZ}$ level of theory, implemented in GAUSSIAN 03 software package. ${ }^{33}$ Here "SDD+Au(2f)" denotes the Stuttgart/ Dresden effective core pseudopotential (ECP) valence basis $^{34,35}$ augmented by two sets of $f$ polarization functions 
(exponents $=1.425,0.468)$. Total energies are given in Table I.

\section{RESULTS AND DISCUSSION}

In Fig. 2, we show histograms of energy distribution for $\mathrm{Au}_{16}, \mathrm{Au}_{17}$, and $\mathrm{Au}_{18}$. It can be seen that for $\mathrm{Au}_{n}$ $(n=16-18)$, there are typically about 40-50 low-lying isomers whose energy is within $0.3 \mathrm{eV}$ from the lowest-lying isomer. However, for $\mathrm{Au}_{19}$, even the energy of the second lowest-lying isomer is already $0.48 \mathrm{eV}$ higher than the lowest-lying isomer. Geometric structures of the low-lying isomers whose energy is within $0.2 \mathrm{eV}$ from the lowest-lying isomer are plotted in Fig. 1. For the leading candidates whose energy is within $0.1 \mathrm{eV}$ from the lowest-lying isomer, their relative energies calculated based on all three basis sets are displayed beneath each isomer (Fig. 1).

Among the low-lying clusters $\mathrm{Au}_{n}(n=15-18)$ displayed in Fig. 1, two generic cage structures can be identified: one is shell-like cage structure and another is spherical-like cage structure. Here we name the former flat cage and the latter hollow cage. Using $\mathrm{Au}_{17 a}$ and $\mathrm{Au}_{17 b}$ as an example (see Fig. 3), we define a flat cage (e.g., $\mathrm{Au}_{17 b}$ ) such that besides the overall shape of the cluster is shell like (or oblate in shape), the $\mathrm{Au}-\mathrm{Au}$ lines (green lines in Fig. 3) connecting through the central region of the cage (in the short-axis direction) are within the range of 3-4.5 $\AA$. Thus, a flat cage may accommodate a small atom such as a hydrogen atom but cannot accommodate another gold atom without major structural distortion. Moreover, we define a hollow cage (e.g., $\mathrm{Au}_{17 a}$ ) such that the $\mathrm{Au}-\mathrm{Au}$ lines (green lines) connecting through the central region of the cage are all greater than $5.2 \AA$. Hence, the hollow cage can accommodate another gold atom.

$\mathbf{A} \mathbf{u}_{15}$. All the five leading candidates for lowest-energy isomers (Fig. 1) exhibit shell-like flat-cage structures. 15a can be viewed as being built upon the lowest-energy structure of $\mathrm{Au}_{14}$, , ,8,11,17,27 also a shell-like shaped isomer. Both $15 a$ and the lowest-energy structure of $\mathrm{Au}_{14}$ possess $C_{2 v}$ symmetry. The energy ranking of the low-lying $\mathrm{Au}_{15}$ isomers is not very sensitive to the basis sets selected. As discussed above, because of the similarity in structures among these candidate low-lying clusters, we expect that the error bar in the DFT relative-energy calculation is relatively small, about the order of $0.1 \mathrm{eV}$. Hence, one of the $15 a, 15 b$, and $15 c$ isomers is likely the global minimum. $15 a$ and $15 b$ can be viewed as isoenergetic because their energy difference is less than $0.06 \mathrm{eV}$. As in the case of anion counterparts, ${ }^{18}$ the flat-cage structures dominate the low-lying population of $\mathrm{Au}_{15}$. It is worthy to note that $15 a, 15 b$, and $15 c$ are also among the top-five lowest-energy anion isomers of $\mathrm{Au}_{15}^{-}$ (Ref. 18).

$\mathbf{A} \mathbf{u}_{16}$. At $n=16$, the anion gold clusters undergo a transition from flat-cage to hollow-cage structure. ${ }^{18}$ Indeed, a large population of low-lying anion isomers of $\mathrm{Au}_{16}^{-}$exhibit hollow cages. In contrast, for neutral $\mathrm{Au}_{16}$, the globalminimum search indicates that the flat-cage structures dominate the low-lying population, similar to the case of $\mathrm{Au}_{15}$. The lowest-energy isomer $16 a$ exhibits $C_{2 v}$ symmetry and is about $0.17 \mathrm{eV}$ lower in energy than the $T_{d}$-symmetry hollowcage $16 j$ (Fig. 1). The latter is a leading candidate for the lowest-energy anion isomer of $\mathrm{Au}_{16}^{-}$(Ref. 18). The second leading candidate for the lowest-energy $\mathrm{Au}_{16}, 16 b$, is merely $0.02 \mathrm{eV}$ higher in energy than $16 a$ at the PBE/DNP level and $0.02 \mathrm{eV}$ lower at the higher level PBEPBE/SDD + Au( $2 f)$. Hence, based on DFT, $16 a$ and $16 b$ can be viewed as isoenergetic. Note that the geometry of $16 b$ is sailing-boatlike, the only non-flat-cage isomer among $16 a-16 e$ (Fig. 1). Since the geometric structure of $16 b$ is drastically different from other flat-cage isomers, high-level $a b$ initio calculation is required to evaluate their relative stability. To this end, we performed single-point energy calculation of $16 a$ and $16 b$ using the resolution-of-the-identity coupled-cluster (RI-CC2) method with the TZVP basis set. The TURBOMOLE software program was used for this independent total-energy calculation. ${ }^{36}$ It is found that the flat-cage structure $16 a$ is $0.68 \mathrm{eV}$ lower in energy than the boatlike structure $16 \mathrm{~b}$. This result shows that the global minimum of $\mathrm{Au}_{16}$ is most likely a flat-cage isomer. The two flat-cage isomer candidates, $16 a$ and $16 c$, hold the highest possibility to be the global minimum, on the basis of DFT calculation with the large basis set (Table I).

$\mathbf{A u}_{17}$. For neutral $\mathrm{Au}_{17}$, the predominant population of low-lying isomers exhibits hollow-cage structures, as in the case of anion $\mathrm{Au}_{17}^{-}$(Ref. 18). Therefore, we can conclude that, for neutral gold clusters, the structural transition from the shell-like flat-cage to spherical-like hollow-cage occurs at $\mathrm{Au}_{17}$. Note that, for anion clusters, the transition is at $\mathrm{Au}_{16}^{-}$. More interestingly, the geometric structure of the lowestlying isomer $17 a$ is identical to that of the lowest-energy isomer of $\mathrm{Au}_{17}^{-}$(Ref. 18). In fact, DFT total-energy calculations with all three basis sets [DNP, LANL2DZ, and SDD $+\mathrm{Au}(2 f)]$ give consistent prediction to the energy ranking (Fig. 1), that is, $17 a$ is the lowest-energy isomer. It appears that $17 a$ is distinctly stable. This distinct stability of $17 a$, either in neutral or in anionic form, is a unique case in the sense that for other gold clusters in the size range of $n$ $=15-18$ the predicted lowest-energy isomer is always more or less sensitive to the selected basis set. $17 a$ processes $C_{2 v}$ symmetry and can be viewed as placing one atom on the top of $16 j$ (Fig. 1). The other two candidates for the lowestenergy isomer of $\mathrm{Au}_{17}, 17 c$ and $17 d$, also exhibit hollowcage structures. However, $17 b$ exhibits a flat-cage structure with $C_{2}$ symmetry. DFT calculation with the larger basis set $[\mathrm{SDD} / \mathrm{Au}(2 f)]$ shows that $17 b$ is about $0.26 \mathrm{eV}$ higher in energy than $17 a$.

$\mathbf{A u}_{18}$. For neutral $\mathrm{Au}_{18}$, again, the hollow-cage structures dominate the population of low-lying isomers. As shown in Fig. 1, all top-five candidate lowest-energy isomers are within $0.08 \mathrm{eV}$ in energy among each other. Because there exist a large number of nearly isoenergetic isomers for $\mathrm{Au}_{18}$, it is not surprising that the energy ranking predicted is sensitive to the selected basis sets (as shown by the relativeenergy values in black, blue and red in Fig. 1). Hence, DFT alone cannot determine the true global minimum of $\mathrm{Au}_{18}$. We can only conclude that the global minimum is likely to be one of the top-five isomers shown in Fig. 1. Note that for anion $\mathrm{Au}_{18}^{-}$, the pyramidal-like isomer $18 i$ (Fig. 1) is also a 
leading candidate for the lowest-energy isomer. ${ }^{18}$ However, in neutral form, $18 i$ is not competitive energetically compared to the hollow-cage isomers $18 a-18 e .18 i$ is about $0.17 \mathrm{eV}$ higher in energy than $18 a$ at the PBE/DNP level, $0.33 \mathrm{eV}$ higher at the PBEPBE/SDD $+\mathrm{Au}(2 f)$ level, and $0.62 \mathrm{eV}$ higher at the RI-CC2/TZVP//PBE/DNP level. The latter relative-energy result clearly shows that the pyramidallike structure is not energetically as favorable as the hollowcage structures for $\mathrm{Au}_{18}$.

$\mathbf{A u}_{19}$. As in the case of anion clusters, ${ }^{18}$ the structural transition from hollow cage to pyramid occurs at $n=19$ due to the overwhelming stability of pyramid $\mathrm{Au}_{20}$ (Ref. 10). Only two low-lying isomers of $\mathrm{Au}_{19}$ are shown in Fig. 1, both exhibiting pyramidal-like structures. $19 a$ corresponds to the removal of a corner atom from the pyramid $\mathrm{Au}_{20}$, while $19 b$, the second lowest-energy isomer, corresponds to the removal of an atom from the edge of the pyramid $\mathrm{Au}_{20}$. Since the perfect pyramid $\mathrm{Au}_{20}$ structure is highly stable (magic-number cluster), a little structural distortion can cause appreciable energy increase. Indeed, $19 b$ is $0.48 \mathrm{eV}$ higher in energy than $19 a$, even though both isomers can be derived by removing only one atom from the pyramid $\mathrm{Au}_{20}$.

\section{CONCLUSION}

On the basis of a global-minimum search by means of combined basin-hopping/DFT method we obtained a large population of low-lying neutral gold clusters in the size range of $n=15-18$, from which we identified several leading candidates for the lowest-energy cluster, including four for $\mathrm{Au}_{15}$, two for $\mathrm{Au}_{16}$, three for $\mathrm{Au}_{17}$, five for $\mathrm{Au}_{18}$, and one for $\mathrm{Au}_{19}$. For $\mathrm{Au}_{15}$ and $\mathrm{Au}_{16}$ it is found that the shell-like flatcage clusters dominate the population of low-lying clusters, while for $\mathrm{Au}_{17}$ and $\mathrm{Au}_{18}$ hollow-cage clusters dominate the low-lying population. Hence, the transition from flat-cage to hollow-cage structure is likely to occur at $\mathrm{Au}_{17}$. In contrast, for the anion counterparts, the structural transition occurs at $\mathrm{Au}_{16}^{-}$. Similar to the anion clusters, the transition from hollow cage to pyramid occurs at $\mathrm{Au}_{19}$. The pyramid $\mathrm{Au}_{19}(19 a)$ is much lower in energy than other low-lying isomers, and thus may be also considered as a magic-number cluster. It is also worthy to mention that the hollow-cage structure $17 a$ (with $C_{2 v}$ point-group symmetry) shows distinct stability, either in neutral or in anionic form, compared to other lowlying isomers. This is a unique case because for other size of clusters considered here (except 19a) the predicted lowestenergy structure is always more or less sensitive to the selected basis sets, but $17 a$ is not. The distinct stability of the hollow-cage $17 a$ calls for the possibility of synthesizing highly stable endohedral gold clusters $\mathrm{M} @ \mathrm{Au}_{17}$ where $\mathrm{M}$, for example, can be metal elements in group I. As such, the bimetallic core/shell clusters are not only closed-shell clusters but also satisfy the 18 -electron rule, ${ }^{37}$ a key factor responsible to the high stability of known gold-based bimetallic clusters. ${ }^{30,38-40}$

\section{ACKNOWLEDGMENTS}

We are grateful to valuable discussions with Professor L.-S. Wang, Professor Pyykkö, Professor J. Zhao, and Dr. X.
Li. This work is supported by grants from the DOE (DEFG02-04ER46164), NSF (CHE and MRSEC), the Nebraska Research Initiative, and by John Simon Guggenheim Foundation and the Research Computing Facility at the University of Nebraska-Lincoln.

${ }^{1}$ R. L. Whetten, J. T. Khoury, M. M. Alvarez, S. Murthy, I. Vez-mar, Z. L. Wang, P. W. Stephens, C. L. Cleveland, W. D. Ludedtke, and U. Landman, Adv. Mater. (Weinheim, Ger.) 5, 8 (1996).

${ }^{2}$ C. A. Mirkin, R. L. Letsinger, R. C. Mucic, and J. Storhoff, Nature (London) 382, 607 (1996).

${ }^{3}$ A. P. Alivisatos, K. P. Johnsson, X. Peng, T. E. Wilson, C. J. Loweth, M. P. Bruchez, and P. G. Schultz, Nature (London) 382, 609 (1996).

${ }^{4}$ P. Pyykkö, Angew. Chem., Int. Ed. 43, 4412 (2004).

${ }^{5}$ P. Schwerdtfeger, Angew. Chem., Int. Ed. 42, 1982 (2003).

${ }^{6}$ H. Häkkinen and U. Landman, Phys. Rev. B 62, R2287 (2000).

${ }^{7}$ J. Wang, G. H. Wang, and J. Zhao, Phys. Rev. B 66, 035418 (2002).

${ }^{8}$ H. Häkkinen, B. Yoon, U. Landman, X. Li, H.-J. Zhai, and L.-S. Wang, J. Phys. Chem. A 107, 6168 (2003).

${ }^{9}$ H. M. Lee, M. Ge, B. R. Sahu, P. Tarakeshwar, and K. S. Kim, J. Phys. Chem. B 107, 9994 (2003).

${ }^{10}$ J. Li, X. Li, H.-J. Zhai, and L.-S. Wang, Science 299, 864 (2003).

${ }^{11}$ L. Xiao and L. Wang, Chem. Phys. Lett. 392, 452 (2004).

${ }^{12}$ F. Remacle and E. S. Kryachko, J. Chem. Phys. 122, 044304 (2005).

${ }^{13}$ R. M. Olson, S. Varganov, M. S. Gordon, H. Metiu, S. Chretien, P. Piecuch, K. Kowalski, S. A. Kucharski, and M. Musial, J. Am. Chem. Soc. 127, 1049 (2005).

${ }^{14}$ Y.-K. Han, J. Chem. Phys. 124, 024316 (2006).

${ }^{15}$ A. V. Walker, J. Chem. Phys. 122, 94310 (2005).

${ }^{16}$ W. Fa, C. Luo, and J. Dong, Phys. Rev. B 72, 205428 (2005).

${ }^{17}$ Y. Gao, S. Bulusu, N. Shao, and X. C. Zeng (unpublished).

${ }^{18}$ S. Bulusu, X. Li, L.-S. Wang, and X. C. Zeng, Proc. Natl. Acad. Sci. U.S.A. 103, 8326 (2006).

${ }^{19}$ M. Kabir, A. Mookerjee, and A. K. Bhattacharya, Phys. Rev. A 69, 043203 (2004).

${ }^{20}$ G. H. Guvelioglu, P. Ma, X. He, R. C. Forrey, and H. Cheng, Phys. Rev. Lett. 94, 026103 (2005).

${ }^{21}$ M. Yang, K. A. Jackson, C. Koehler, Th. Frauenheim, and J. Jellinek, J. Chem. Phys. 124, 024308 (2006).

${ }^{22}$ R. Fournier, J. Chem. Phys. 115, 2165 (2001).

${ }^{23}$ J. Zhao, Y. Luo, and G. Wang, Eur. Phys. J. D 14, 309 (2001).

${ }^{24}$ E. M. Fernández, J. M. Soler, I. L. Garzón, and L. C. Balbás, Phys. Rev. B 70, 165403 (2004); E. M. Fernández, J. M. Soler, and L. C. Balbás, ibid. 73, 235433 (2006).

${ }^{25}$ J. P. K. Doye and D. J. Wales, New J. Chem. 773 (1998).

${ }^{26}$ I. L. Garzón, K. Michaelian, M. R. Beltrán, A. Posada-Amarillas, P. Ordejón, E. Artacho, D. Sánchez-Portal, and J. M. Soler, Phys. Rev. Lett. 81, 1600 (1998); K. Michaelian, N. Rendón, and I. L. Garzón, Phys. Rev. B 60, 2000 (1999).

${ }^{27}$ F. Furche, R. Ahlrichs, P. Weis, C. Jacob, S. Glib, T. Bierweiler, and M. M. Kappes, J. Chem. Phys. 117, 6982 (2002).

${ }^{28}$ D. J. Wales and H. A. Scheraga, Science 285, 1368 (1999).

${ }^{29}$ S. Yoo and X. C. Zeng, Angew. Chem., Int. Ed. 44, 1491 (2005).

${ }^{30}$ Y. Gao, S. Bulusu, and X. C. Zeng, J. Am. Chem. Soc. 127, 15680 (2005).

${ }^{31}$ J. P. Perdew, K. Burke, and M. Ernzerhof, Phys. Rev. Lett. 77, 3865 (1996).

${ }^{32}$ B. Delley, J. Chem. Phys. 92, 508 (1990).

${ }^{33}$ M. J. Frisch, G. W. Trucks, H. B. Schlegel et al., GaUSSIAN 03, Revision C.02, Gaussian, Inc., Pittsburgh, PA, 2003.

${ }^{34}$ M. Dolg, U. Wedig, H. Stoll, and H. Preuss, J. Chem. Phys. 86, 866 (1987).

${ }^{35}$ P. Schwerdtfeger, M. Dolg, W. H. E. Schwarz, G. A. Bowmaker, and P. D. W. Boyd, J. Chem. Phys. 91, 1762 (1989).

${ }^{36}$ R. Ahlrichs, M. Bär, H.-P. Baron et al., tURBOMOLE v5-8-0.

${ }^{37}$ P. Pyykkö, J. Organomet. Chem. (to be published).

${ }^{38}$ P. Pyykkö and N. Runeberg, Angew. Chem., Int. Ed. 41, 2174 (2002).

${ }^{39}$ X. Li, B. Kiran, J. Li, H. J. Zhai, and L.-S. Wang, Angew. Chem., Int. Ed. 41, 4786 (2002).

${ }^{40}$ Y. Gao, S. Bulusu, and X. C. Zeng, ChemPhysChem (to be published). 